\title{
DISTORTION THEOREMS IN THE THEORY OF SCHLICHT FUNCTIONS
}

\author{
SHOHEI NAGURA and YUSAKU KOMATU
}

1. Let $\subseteq$ be the family of analytic functions $F(z)$ which are regular and schlicht in the circle $E:|z|<1$ and normalized at the origin such that $F(0)=0$ and $F^{\prime}(0)=1$. Let $\mathfrak{A}$ be the subfamily of $\mathfrak{S}$ consisting of all functions, each of which possesses, as boundary of its image-domain, a closed Jordan curve which is supposed to be regular analytic. Then $\mathfrak{A}$ is everywhere dense in the original family $\mathfrak{\Im}$. Hence, by an approximation theorem of Carathéodory on the kernel of sequence of domains, we may restrict ourselves within $\mathfrak{A}$, so far as we concern the estimation of continuous functionals on $\Im$. We know the following fundamental theorem due to Schaeffer-Spencer ${ }^{1)}$ :

THEOREM 1. Let $F(z)$ be any function of $\mathfrak{A}$ and $e^{t_{0}}$ be any positive number greater than the maximum modulus of $F(z)$ on $|z|=1$. Then there exists a oneparameter family of functions $h(z, t)$, defined for $z \in E$ and $0 \leqq t \leqq t_{0}$, satisfying the differential equation

$$
\frac{\partial h(z, \underline{t})}{\partial t}=z p(z, t) \frac{\partial h(z, t)}{\partial z}
$$

with boundary conditions $h\left(z, t_{0}\right)=z$ and $h(z, 0)=f(z) \equiv e^{-t_{0}} F(z)$. Here $p(z, t)$ is, for each $t\left(0 \leqq t \leqq t_{0}\right)$, an analytic function regular and with positive real part with respect to $z \in E$ and moreover satisfying the condition $p(0, t)=1$.

Let now $R$ denote the (doubly-connected) ring domain on $w$-plane whose boundary consists of $|w|=1$ and of the image-curve of $|z|=1$ by the mapping $w=e^{-t_{0}} F(z)$. Then, each function $w=h(z, t)$ may be regarded as the mapping function from $E$ onto a domain bounded by a level curve of harmonic measure of $|w|=1$ with respect to $R$, satisfying the following conditions :

$$
h(0, t)=0, \quad h^{\prime}(0, t) \equiv\left[\frac{\partial h(z, t)}{\partial z}\right]^{z=0}=e^{t-i_{0}} .
$$

Hence, $e^{t_{0}-t} h(z, t)$ belongs, for each $t\left(0 \leqq t \leqq t_{0}\right)$, to the family $\mathfrak{A}$. Now, we introduce a new family $\{f(z, t)\}$ by means of the relation

Received February 3, 1950.

1) A. C. Schaeffer and D. C. Spencer, The coefficients of schlicht functions, II. Duke Math. Journ. 12 (1945), 107-125. 


$$
f(z) \equiv h(f(z, t), t) .
$$

If we denote by $D$ the image-domain of $E$ by the mapping $w=f(z)$, then $w$ $=f(z, t)$ maps $E$ onto the domain $D_{t}$ corresponding to $D$ which are now considered to be laid on the $\zeta$-plane, by the mapping $\zeta=h(w, t)$, i.e., $D=h\left(D_{t}, t\right)$. Since each $f(z, t)$ maps $E$ onto a domain bounded by a regular Jordan curve and satisfies $f(0, t)=0, f^{\prime}(0, t)=e^{-t}$, the funciton $e^{t} f(z, t)$ belongs, for each $t\left(0 \leqq t \leqq t_{0}\right)$, to the family $\mathfrak{A}$. The differential equation for this family $\{f(z, t)\}$ is immediately obtained from the preceding theorem. ${ }^{2,31}$

THEOREM 2. Under the same conditions as in the preceding theorem, the function $f(z) \equiv e^{-t_{0}} F(z)$ is delermined as the integral $f(z)=f\left(z, t_{0}\right)$ of the differential equation

$$
\frac{\partial f(z, t)}{\partial t}=-p(f(z, t), t) f(z, t)
$$

with initial condition $f(z, 0)=z$.

Making use of the last theorem, we shall show in this Note that various distortion theorems in the theory of $\subseteq$ can also be proved by the differential equation (1.2). The procedure is, however, analogous with that using the Löwner's differential equation for slit mapping. The last part of the Note will be devoted to a generalization of the method to doubly-connected case.

2. On account of the above-mentioned properties of $p(z, t)$, it can be expressed by the so-called Herglotz's formula, that is,

$$
p(w, t)=\int_{0}^{2 \pi} \frac{e^{i \theta}+w}{e^{i \theta}-w} d \tau(\theta, t) \quad(|w|<1)
$$

where $d \tau(\theta, t)$ is the differential with respect to $\theta$ and, for each $t\left(0 \leqq t \leqq t_{0}\right)$,

$$
\tau(\theta, t)=\frac{1}{2 \pi} \int_{0}^{\theta} \Re p\left(e^{i \varphi}, t\right) d \varphi
$$

is an increasing function of $\theta$ such that $\tau(0, t)=0$ and $\tau(2 \pi, t)=1$. Hence we have the Harnack's inequalities

$$
\frac{1-|w|}{1+|w|} \leqq \Re p(w, t) \leqq|p(w, t)| \leqq \frac{1+|w|}{1-|w|} \quad(|w|<1) .
$$

Using these inequalities, we shall first prove, as an example, a famous distortion theorem of Koebe-Bieberbach : ${ }^{4)}$

2) Y. Komatu and S. Nagura, Theory of schlicht functions. Sûgaku 1 (1948/9), 286-302. (Japanese.)

3) Y. Komatu, Fundamental differential equations in the theory of conformal mapping. Proc. of Japan Acad. Tokyo 25 (1949), 1-10.

4) A proof of the theorem by using Löwner's equation has been given by G. M. Golusin, Über einige Abschätzungen von Funktionen, welche den Kreis konform und schlicht abbilden. Recueil Math. 36 (1929), 152-172. (in Russian.) 
Theorem 3. For any function $F(z) \in \mathbb{S}$, we have

$$
\frac{|z|}{(1+|z|)^{2}} \leqq|F(z)| \leqq \frac{|z|}{(1-|z|)^{2}} \quad(|z|<1) .
$$

Each equality holds, at a certain point $z_{0}\left(0<\left|z_{0}\right|<1\right)$, only for the function

$$
F(z)=\frac{z}{(1+\varepsilon z)^{2}}, \quad \varepsilon= \pm \frac{\left|z_{0}\right|}{z_{0}} .
$$

Proof. It is sufficient to prove (2.3) for functions $F(z)=e^{t_{0}} f(z)$ belonging to $\mathfrak{A}$. Supposing that $z$ is an arbitrarily fixed point in $E$, we may write $d / d t$ instead of $\partial / \partial t$. We can then rewrite (1.2) in the form

$$
\frac{d \lg w}{d t}=-p(w, t) \quad\left(w=w_{t} \equiv f(z, t)\right) .
$$

Comparing here the real parts of both sides we have

$$
\frac{d \lg |w|}{d t}=-\Re p(w, t)
$$

and hence, by Harnack's inequalities (2.2),

$$
\frac{1-|w|}{1+|w|} \leqq-\frac{d \lg |w|}{d t} \leqq \frac{1+|w|}{1-|w|} \text {. }
$$

Integrating each of these differential inequalities between the range $0 \leqq t \leqq t_{0}$ and hence $\left|w_{0}\right| \supseteqq\left|w_{t}\right| \triangleq\left|w_{t_{0}}\right|$ with $w_{0}=f(z, 0)=z, w_{t_{0}}=f\left(z, t_{0}\right)$, we obtain

$$
\left(1+\left|f\left(z, t_{0}\right)\right|^{2}\right) \frac{|z|}{(1+|z|)^{2}} \leqq e^{t_{0}}|f(z, t)| \leqq\left(1-\left|f\left(z, t_{0}\right)\right|^{2}\right) \frac{|z|}{(1-|z|)^{2}},
$$

from which the relation (2.3) follows immediately. Each equality in (2.3) holds only in the limiting case where $t_{0}$ tends to infinity and $p(w, t)$ tends to $(1+\varepsilon w) /(1-\varepsilon w)$, whence we get the extremal function:

$$
e^{t_{0}} f\left(z, t_{0}\right)=\left(1+\varepsilon f\left(z, t_{0}\right)\right)^{2} \frac{z}{(1+\varepsilon z)^{2}} \rightarrow \frac{z}{(1+\varepsilon z)^{2}} \quad\left(t_{0} \rightarrow \infty\right) .
$$

We shall next prove, as second typical example, a classical distortion theorem due to $R$. Nevanlinna: ${ }^{51}$

TheOREM 4. For any function $F(z) \in \Im$, we have

$$
\frac{1-|z|}{1+|z|} \leqq\left|\frac{z F^{\prime}(z)}{F(z)}\right| \leqq \frac{1+|z|}{1-} \quad(|z|<1) .
$$

Each equality holds only for the same extremal function as that in the preceding theorem, that is, for (2.4).

Proof. Differentiating both sides of (2.5) with respect to $z$, we get

$$
\frac{d \lg w^{\prime}}{d t}=-p(w, t)-w p^{\prime}(w, t) \quad\left(w^{\prime}=\frac{\partial f(z, t)}{\partial z} \quad p^{\prime}=\frac{\partial p(w, t)}{\partial w}\right),
$$

5) A proof by the method of Löwner's equation has been given by G. M. Golusin, Über die Verzerrungssätze der schlichten konformen Abbildungen. Recueil Math. 1 (43) (1936), 127-135. (in Russian.) 
and hence, by remembering again the equation (2.5), we have

$$
\frac{d}{d t} \lg \frac{w^{\prime}}{w}=-w p^{\prime}(w, t) \text {. }
$$

On the other hand, we have, by differentiating (2.1) with respect to $w$,

$$
p^{\prime}(w, t)=\int_{0}^{2 \pi} \frac{2 e^{i \theta}}{\left(e^{i \theta}-w\right)^{2}} d \tau(\theta, t),
$$

and we get from (2.6), by taking account of (2.1),

$$
d t=\left(\int_{0}^{2 \pi} \frac{1-|w|^{2}}{\left|e^{i \theta}-w\right|^{2}} d \tau(\theta, t)\right)^{-1} \frac{-d|w|}{|w|} .
$$

Combining these relations we obtain a differential inequality

$$
\begin{aligned}
& |d \lg | \frac{w^{\prime}}{w}||=\left|-\Re\left(w p^{\prime}(w, t)\right) d t\right| \\
& \quad=\left|\left(-\int_{0}^{2 \pi} \Re \frac{2 w e^{i \theta}}{\left(e^{i \theta}-w\right)^{2}} d \tau(\theta, t) / \int_{0}^{2 \pi} \frac{1-|w|^{2}}{\left|e^{i \theta}-w\right|^{2}} d \tau(\theta, t)\right) \frac{-d|w|}{|w|}\right| \leqq \frac{-2 d|w|}{1-|w|^{2}},
\end{aligned}
$$

since $d \tau(\theta, t) \triangleq 0$ and $d|w| \leqq 0$. Integrating from 0 to $t_{0}$ with respect to $t$ and putting $f\left(z, t_{0}\right)=e^{t_{0}} F(z)$, we conclude the distortion formula (2.8). As to the equality sign at a point $z_{0}$ with $0<\left|z_{0}\right|<1$, we notice again that it can only occur in the limiting case where $t_{0} \rightarrow \infty$. If we put $f\left(z_{0}, t\right)=\rho\left(z_{0}, t\right) e^{i_{\rho}\left(z_{0}, t\right)}$, it is easily seen that the equality in (2.8) can hold only when

$$
\theta \equiv \varphi+\frac{\pi}{2} \mp \frac{\pi}{2} \quad(\bmod 2 \pi),
$$

in which case $\tau(\theta, t)$ must be a step function whose differential is equal to 0 except for the value just assigned in (2.13). In this limiting case we have $p(w, t)=(1+\varepsilon w) /(1-\varepsilon w)$ with $\varepsilon= \pm\left|z_{0}\right| / z_{0}$ and, hence, obtain the extremal function (2.4).

The inequalities (2.8) can be further sharpened, as shown by Golusin ${ }^{6)}$ by making use of Löwner's equation, to the following form:

$$
\left|\lg \frac{z F^{\prime}(z)}{F(z)}\right| \leqq \lg \frac{1+|z|}{1-|z|} \quad(|z|<1),
$$

which can also be proved by a similar method as that in the last theorem.

We shall mention here the so-called Golusin's rotation theorem. Though almost all the distortion theorems in the theory of $\subseteq$ can be derived by the classical method of Bieberbach's area theorem or the more powerful method due to Grunsky "), the rotation theorem for $\arg F^{\prime}(z)$ can now be proved, in its pre-

6) G. M. Golusin, Ergänzung zur Arbeit “Über die Verzerrungssätze der schlichten konformen Abbildungen." Recueil Math. 2 (44) (1937), 685-688. (in Russian.)

7) H. Grunsky, Neue Abschätzungen zur konformen Abbildung ein- und mehrfach zusammenhängender Bereiche. Schriften math. Sem. u. Inst. angew. Math. Univ. Berlin 1 (1932/3), 95-140. 
cise form, only by the method based upon Löwner's differential equation. Golusin ${ }^{\text {) }}$ has given it a first proof which has been simplified a little by Basilewitsch. ${ }^{91}$ A proof we shall give in the following lines resembles closely to that of Basilewitsch. The theorem states:

THEOREM 5. For any function $F(z) \in \Subset$, we have

$$
\left|\arg F^{\prime}(z)\right| \leqq \begin{cases}4 \arcsin |z| & (|z| \leqq 1 / \sqrt{2}), \\ \pi+\lg \frac{|z|^{2}}{1-|z|^{2}} & (1 / \sqrt{ } 2 \leqq|z|<1),\end{cases}
$$

$\arg F^{\prime}(z)$ denoting here the branch which vanishes at the origin. For any point $z_{0}$ of $E$ there exists an extremal function.

Proof. Comparing the imaginary parts of both sides of (2.9) and then making use of (2.1) and (2.11), we obtain

$$
\frac{d \arg w^{\prime}}{d t}=-\Im\left(\not(w, t)+w p^{\prime}(w, t)\right)=\int_{0}^{2 \pi} \frac{2 \Im\left(1-e^{-i 0} w\right)^{2}}{\left|e^{i \theta}-w\right|^{4}} d \tau(\theta, t),
$$

which becomes, by substituting (2.12),

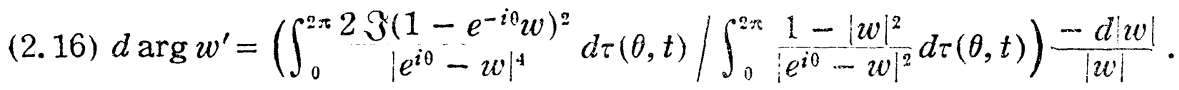

Since $\tau(\theta, t)$ is increasing, i.e., $d \tau(\theta, t) \geqslant 0$, we get

$$
\left|d \arg w^{\prime}\right| \leqq \operatorname{Max}_{0 \leqq 0 \leqq 2 \pi} \frac{2\left|3\left(1-e^{-i \theta} w\right)^{2}\right|}{\left|e^{i \theta}-w\right|^{2}} \frac{-2 d|w|}{|w|\left(1-|w|^{2}\right)}
$$

$$
\leqq \begin{cases}\frac{-4 d|w|}{\sqrt{1-|w|^{2}}} & \left(|w| \leqq \frac{1}{\sqrt{2}}\right) \\ \frac{-2 d|w|}{|w|\left(1-|w|^{2}\right)} & \left(\frac{1}{\sqrt{2} \leqq|w|<1)},\right.\end{cases}
$$

from which we conclude, by integration,

$$
\left|\arg f^{\prime}\left(z, t_{0}\right)\right| \leqq\left\{\begin{array}{l}
4 \arcsin |z|-4 \arcsin \left|f\left(z, t_{0}\right)\right| \quad\left(|z| \leqq \frac{1}{\sqrt{2}}\right), \\
\pi-4 \arcsin \operatorname{Min}\left(\left|f\left(z, t_{0}\right)\right|,-\frac{1}{\sqrt{2}}\right)+\lg \frac{|z|^{2}}{1-|z|^{2}}\left(\frac{1}{\sqrt{2}} \leqq|z|<1\right) .
\end{array}\right.
$$

As $\arg F^{\prime}(z)=\arg f^{\prime}\left(z, t_{0}\right)$, the desired relation (2.15) follows immediately. We now examine the extremal case at $z_{0} \in E$. As is seen from (2.18), the equality in (2.15) can hold only for the limiting case $t_{0} \rightarrow \infty$. If we regard (2.15) as double inequalities for the quantity $\arg F^{\prime}(z)$ itseif, the lower or upper bound is reached only when $d r(\theta, t)$ is equal to 0 except for a value of $\theta$ given by

8) G. M. Golusin, loc. cit. ${ }^{5)}$ and Sur les théorèmes de rotation dans la théorie des fonctions univalentes. Recueil Math. 1 (43)(1936), 293-296.

9) J. Basilewitsch, Sur les théorèmes de Koebe-Bieberbach. Recueil Math. 1 (43) (1936), 283-292. 


$$
\frac{2 \Im\left(1-\rho e^{i(p-\theta)}\right)^{2}}{\left|1-\rho e^{i(p-\theta)}\right|^{2}}= \begin{cases}\mp 2 \rho \sqrt{1-\rho^{2}} & (\rho \leqq 1 / \sqrt{2}), \\ \mp 1 & (1 / \sqrt{2} \leqq \rho<1)\end{cases}
$$

respectively, where we put for brevity $f\left(z_{0}, t\right)=\rho e^{i \rho}=\rho\left(z_{0}, t\right) e^{i\left(z_{0}, t\right)}$. The last equation can also be written in the form

$$
\rho e^{i(\rho-\theta)}= \begin{cases}\rho\left(\rho \pm i \sqrt{1-\rho^{2}}\right) & (\rho \leqq 1 / \sqrt{2}), \\ 1+\eta \sqrt{2 \rho^{2}-1} \pm i\left(1-\eta \sqrt{2 \rho^{2}-1}\right) & (1 / \sqrt{2} \leqq \rho<1)\end{cases}
$$

herein $\eta$ may be equal to either +1 or -1 . After substituting this expression for $\rho e^{i(p-\theta)}$ in the right-hand side, the integration of the equation

$$
\frac{d \rho}{d t}=-\rho \frac{1-\rho^{2}}{\left|1-\rho e^{i(p-\theta)}\right|^{2}} \quad\left(\rho\left(z_{0}, 0\right)=\left|z_{0}\right|\right)
$$

yields $\rho\left(z_{0}, t\right)$ as a monotone-decreasing continuous function of $t$, and then, again by (2.19), the quantity $\rho e^{i(p-\theta)}$ is itself determined as a function of $t$. But for the step function $\tau(\theta, t)$, we get $p(w, t)=\left(e^{i \theta}+w\right) /\left(e^{i \theta}-w\right)\left(w=f\left(z_{0}, t\right)\right)$ and hence, by (2.5),

$$
\frac{d \varphi}{d t}=-\Im p(w, t)=-\frac{2 \Re\left(\rho e^{-i(p-\theta)}\right)}{\left|1-\rho e^{i(p-\theta)}\right|^{2}} \quad\left(\varphi\left(z_{0}, 0\right)=\arg z_{0}\right) .
$$

Since the quantity $\rho e^{i(p-\theta)}$ has already been determined as a function of $t$, the integration of the last equation determines $\varphi=\varphi\left(z_{0}, t\right)$, and hence also $\theta=\theta\left(z_{0}, t\right)$, as a function of $t$. Therefore, the Poisson kernel $p\left(\rho e^{i,}, t\right)=\left(1+\rho e^{i \phi}\right) /\left(1-\rho e^{i \rho}\right)$ is also determined as a function of $t$ for which we have, as can be seen from the procedure stated above, the limit relations

$$
\lim _{t \rightarrow \infty} \arg f^{\prime}\left(z_{0}, t\right)= \begin{cases}\mp 4 \arcsin |z| & \left(\left|z_{0}\right| \leqq 1 / \sqrt{2}\right), \\ \pm\left(\pi+\lg \frac{1-\left|z_{0}\right|^{2}}{\left|z_{0}\right|^{2}}\right) & \left(1 / \sqrt{2} \leqq\left|z_{0}\right|<1\right) .\end{cases}
$$

This completes the proof of the theorem.

The other various distortion or rotation theorems of $\subseteq$ may also be proved in quite similar manner.

3. Corresponding to two theorems stated in the first paragraph, the fundamental differential equations can also be derived for doubly-connected case. ${ }^{10}$ r Let now $D$ be a ring domain on the $w$-plane whose boundary is composed of $|w|=1$ and of a closed curve $C$ surrounding the unit circle in its interior which may be, for simplicity, supposed to be regular analytic. Let the modulus (the essentially unique conformal invariant) of $D$ be $\lg Q^{-1}(0<Q<1)$, and let $w$ $=F(z)$ with $F(1)=1$ map $1<|z|<Q^{-1}$ onto $D$. Let $Q_{0}$ be any positive number less than unity such that the closed domain $D+C$ is contained in the annular

10) See loc. cit. ${ }^{3}$ ) 
let $V$ be a neighbourhood of $G / N$ having no non-trivial subgroup. Then there exists an neighbourhood $W$ of $G$ such that $f(W) \subset V$ and that $W \cap N$ contains no non-trivial subgroup. It is clear that the only subgroup in $W$ is the identity group.

Proof of the theorem. First we consider some special cases.

i) Let $L$ be discrete. In this case $G$ and $G / L$ are locally isomorphic. Hence the assertion is obvious.

ii) Let $L$ be a connected semi-simple Lie group with the center $\varepsilon$. Let $A(L)$ be the group of all continuous isomorphisms of $L$, and $I(L)$ the subgroup composed of inner automorphisms. It is well-known that $A(L)$ is a linear Lic group, and $I(L)$ coincides with the identity component of $A(L)$.

Now let $g$ be an element of $G$. Putting

$$
\delta(g) l=g^{-1} l g \text { for } l \in L,
$$

we obtain a continuous homomorphism $\delta$ of $G$ into $A(L)$. Denote by $C$ the kernel of the homomorphism: $C=\{c ; l c=c l$, for $l \in L\}$. Next let $\tilde{\delta}(g)$ be the coset of $A(L)$ mod. $I(L)$ containing $\delta(g)$. Then $\tilde{\delta}$ gives a continuous homomorphism of $G$ into $A(L) / I(L)$. Let $N$ be the kenrel of $\tilde{\delta}_{\text {. }}$.

Because $A(L) / I(L)$ is discrete, $N$ is an open subgroup in $G_{\text {。 }}$ Now every element of $N$ induces an inner automorphism of $L$. Hence $N=C L$. On the other hand as the center of $L$ is $e, C \cap L=e$, whence $N=C \times L$. Thus the isomorphism $N / L \cong C$ and the openness of $N$ imply our assertion.

iii) Let $L$ be a connected commutative Lie group. Denote by $N$ the centralizer of $L: N=\{g ; l g=g l$, for $l \notin L\}$. By a similar argument as above $C / N$ is a Lie group.

Now let $Z$ be the center of $N$. Then by Lemma $1 N / Z$ has no small subgroup. Now, because $Z$ has no small subgroup and is commutative, $Z$ is a Lie group. Hence $Z / L$ is a Lie group. Thus by using LEMma 2 twice we have the desired proposition.

iv) General case. Let $L_{1}$ be the identity component of $L$, and $L_{2}$ the largest solvable invariant subgroup of $L_{1}$. And let $L_{s}$ be the identity component of $L_{2}$. Denote by $L_{4}$ the topological commutator subgroup of $L_{3}, L_{5}$ the topological commutator subgroup of $L_{4}$, and so on. Then we get $a$ sequence

$$
L_{0}=L \supset L_{1} \supset L_{2} \supset \ldots \supset L_{n} \supset L_{n+1}=e
$$

of characteristic subgroups of $L$ such that every $L_{i} / L_{i+1}$ is either discrete, connected commutative, or connected semi-simple with no center. Considering $G / L_{1}, G / L_{2}, \ldots$, in order, we get the result in virtue of above i), ii) and iii). Q.E.D. 
dure is, however, also analogous with that using the differential equation for slit mappings. ${ }^{12)}$

THEOREM 8. Any function $w=F(z)$ with $F(1)=1$, mapping $1<|z|<R$ onto a ring domain $D$ bounded by $|w|=1$ and contained in $1<|w|<M$, satisfies the distortion inequalities

$$
\Phi_{M}(|z|) \leqq|F(z)| \leqq \Phi_{M}(-|z|) \quad(1<|z|<R) ;
$$

$w=\emptyset_{M}(z)$ denoting the function which maps $1<|z|<R$ onto the ring $1<|w|$ $<M$ cut along a rectilinear segment on real axis starting from $w=M$, such that $\Phi_{M}(1)=1$. Each equality in (3.5) holds, at any given point $z_{0}$ in $1<|z|<R$, only for the function

$$
F(z)=\bar{\varepsilon} \Phi_{M}(\varepsilon z) \quad\left(\varepsilon= \pm \frac{\left|z_{0}\right|}{z_{0}}\right) .
$$

Proof. It is sufficient to prove (3.5) for such functions $F(z)$ as in the previous theorem with $Q^{-1}=R, Q_{J}^{-1}=M$. Supposing that $z$ is an arbitrarily fixed point in $1<|z|<R$, we may write $d / d \lg q$ in place of $\partial / \partial \lg q$, and hence the equation (3.4) may be written in the form:

$$
\frac{d \lg w}{d \lg q}=L(w, q) \quad\left(w=w_{q} \equiv F(z, q)\right) .
$$

Comparing the real parts of both sides we have, by (3.2),

$$
\frac{d \lg w}{d \lg q}=\Re L(w, q)=2 \int_{0}^{2 \pi} \Re A\left(w, q^{-1} e^{i \theta} ; q\right) d \tau(\theta, q) .
$$

Now, it can be shown that

$$
\Re A\left(w, q^{-1} e^{i \theta} ; q\right)=\sum_{n=1}^{\infty} \frac{q^{n}}{1-q^{2 n}}\left(|w|^{n}-\frac{1}{|w|^{n}}\right) \cos n(\theta-\varphi) \quad\left(w=|w| e^{i, \rho}\right)
$$

is an even function of $\theta-\varphi$ which, for $|w|>1$ and $0<q<1$, decreases strictly monotonic as $\theta-\varphi$ varies from 0 to $\pi .^{13)}$ We have hence the following differential inequalities:

$$
2 \Re A\left(-|w|, q^{-1} ; q\right) \leqq \frac{d \lg |w|}{d \lg q} \leqq 2 \Re A\left(|w|, q^{-1} ; q\right) .
$$

The differential equations

$$
\frac{d \lg |w|}{d \lg q}=2 \Re A\left(\mp|w|, q^{-1} ; q\right) \quad\left(R^{-1} \geqq q \geqq M^{-1}\right),
$$

integrated with common initial condition: $w=z$ for $q=R^{-1}$, yield for $q=M^{-1}$ the absolute values of extremal mapping function, i.e., $|w|=\Phi_{M}(\mp|z|)$. Since

12) Cf. Y. Komatu, Untersuchungen über konformen Abbildung von zweifach zusammenhängenden Gebieten. Proc. Phys.-Math. Soc. 25 (1943), 1-42, where the concrete expressions for various quantities, especially for extremal functions, are also given.

13) Cf. loc. cit. ${ }^{12)}$, p. 26 et seq. 
$d \lg q<0$, we get in fact the desired inequalities (3.5). The last fart of the theorem concerning the equality sign is also immediate.

If we let the bound $M$ tend to infinity in the last theorem, we obtain the distortion theorem for general function $w=F(z)$ which maps $1<|z|<R$ onto a ring domain bounded by $|w|=1$ and contained outside the unit circumference; that is, we have the distortion formulae: ${ }^{14}$

$$
\Phi(|z|) \leqq|F(z)| \leqq \Phi(-|z|) \quad \text { with } \quad \Phi(z)=\lim _{M \rightarrow \infty} \Phi_{M}(z)
$$

Mathematical Institute, Nagoya University

Tokyo Institute of Technology

14) Cf. also loc. cit. ${ }^{12)}$. 